\title{
Methodology of the Access to Care and Timing Simulation Model for Traumatic Spinal Cord Injury Care
}

\author{
Argelio Santos, Nader Fallah, Rachel Lewis, Marcel F. Dvorak, Michael G. Fehlings, \\ Anthony S. Burns, ${ }^{5}$ Vanessa K. Noonan,' Christiana L. Cheng, Elaine Chan, \\ Anoushka Singh, ${ }^{6}$ Lise Bélanger, Derek Atkins ${ }^{2}$ and the RHSCIR Network
}

\begin{abstract}
Despite the relatively low incidence of traumatic spinal cord injury (tSCI), the management and care of persons with tSCI can be resource intensive and complex, spanning multiple phases of care and disciplines. Using a simulation model built with a system level view of the healthcare system allows for prediction of the impact of interventions on patient and system outcomes from injury through to community reintegration after tSCI. As has been previously described, the Access to Care and Timing (ACT) project developed a simulation model for tSCI care using techniques from operations research. The objective of this article is to briefly describe the methodology and the application of the ACT Model, as it was used in several of the articles in this focus issue. The approaches employed in this model provide a framework to look into the complexity of interactions both within and among the different SCI programs, sites, and phases of care.
\end{abstract}

Keywords: health progression model; operations research; processes of care; simulation model; spinal cord injury

\section{Introduction}

D YNAMIC SIMULATION MODELING can assist in answering complex problems faced by healthcare systems. Simulations can be utilized to assess the impacts of different "what if" scenarios, circumventing the need for resource intensive experimentation. For example, the World Health Organization (WHO) included modeling to assist in providing recommendations in the 2013 guidelines for the use of antiretroviral therapy in human immunodeficiency virus infection, and "Whole Disease Modeling" has been developed to provide a system level framework to inform resource allocation in cancer. ${ }^{1-3}$ To support the rapidly growing application of simulation modeling in healthcare, the International Society for Pharmacoeconomics and Outcomes Research (ISPOR) Dynamic Simulation Modeling Emerging Good Practices Task Force has recently outlined guidelines to facilitate consistency between methodology, publications, and transparency. ${ }^{4,5}$

The objective of this article is to briefly describe the methodology used to create the Access to Care and Timing (ACT) simulation model (ACT Model V1.0) for traumatic spinal cord injury $(\mathrm{tSCI})$ care as it was used in several of the articles in this focus issue. The development of the ACT Model has been described elsewhere. $^{6-8}$ The ACT Model encompasses a Processes of Care Model (PCM), a Health Progression Model (HPM), and an Incidence Forecasting Model (IFM) (Fig. 1). The PCM was developed using operations research methodologies, mainly Discrete Event Simulation (DES), to model the processes of care in the acute and rehabilitation phases. The HPM tracks long-term health and cost outcomes, while the IFM predicts the future incidence of tSCI, for which its implication on healthcare resource needs are reported in an article by Ahn and colleagues in this focus issue.

Despite the relatively low incidence, the management and care of persons with tSCI can be resource intensive and complex, spanning multiple phases of care and disciplines. Using a simulation model built with a system level view of the healthcare system allows for prediction of the impact of interventions on patient and system outcomes from injury through to community reintegration after tSCI. The model is a tool for healthcare administrators, clinicians, and researchers to examine the impact of interventions on patient and system level outcomes based on the following: a) patient characteristics; b) injury characteristics; c) interventions; d) resource availability; and e) decision protocols.

\footnotetext{
${ }^{1}$ Rick Hansen Institute, Vancouver, British Columbia, Canada.

${ }^{2}$ Sauder School of Business, ${ }^{3}$ Department of Orthopedics, University of British Columbia, Vancouver, British Columbia, Canada.

${ }^{4}$ Department of Surgery, ${ }^{5}$ Department of Medicine, University of Toronto, Toronto, Ontario, Canada.

${ }^{6}$ SCI Clinical Research Unit, Toronto Western Hospital, Toronto, Ontario, Canada.

${ }^{7}$ Acute Spine Unit, Vancouver General Hospital, Vancouver, British Columbia, Canada.
}

(c) Argelio Santos et al., 2017; Published by Mary Ann Liebert, Inc. This Open Access article is distributed under the terms of the Creative Commons Attribution Noncommercial License (http://creativecommons.org/licenses/by-nc/4.0/) which permits any noncommercial use, distribution, and reproduction in any medium, provided the original author(s) and the source are credited. 


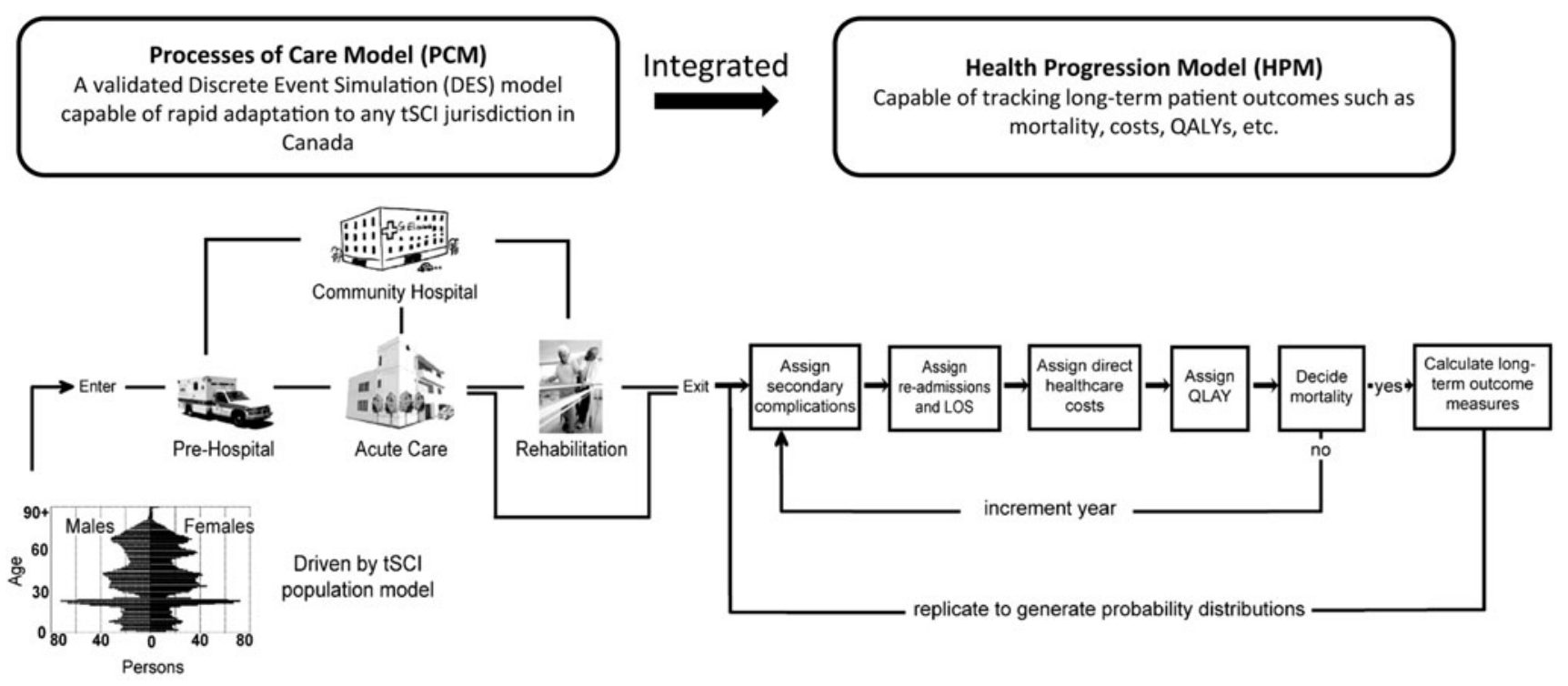

Incidence Forecasting Model (IFM)

Predicts future incidence of $\mathrm{tSCl}$ using population projection and $\mathrm{tSCl}$ incidence data

FIG. 1. Integration of the Processes of Care Model (PCM) and the Health Progression Model (HPM) in the Access to Care and Timing (ACT) simulation model of traumatic spinal cord injury (tSCI). Simulation can be driven by existing tSCI incidence data or by incidence predicted from the Incidence Forecasting Model (IFM). QALYs, quality adjusted life-years; LOS, length of stay. Adapted and reprinted with permission from Noonan and colleagues, ${ }^{6}$ and adapted with permission from Atkins and colleagues. ${ }^{7}$

\begin{tabular}{|c|c|c|c|c|c|c|c|c|c|c|c|c|c|c|c|c|}
\hline & 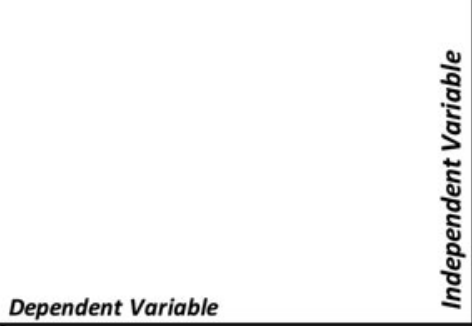 & 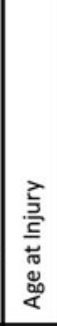 & 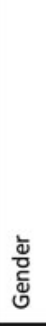 & 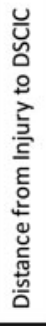 & 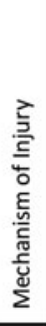 & 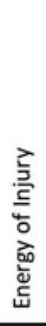 & 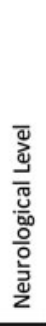 & 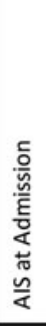 & $\begin{array}{l}\text { U. } \\
\text { O } \\
\text { \& } \\
\text { ¿ } \\
\end{array}$ & 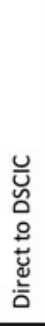 & 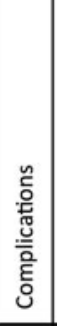 & 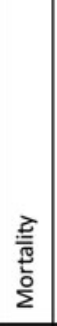 & 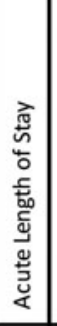 & 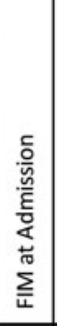 & 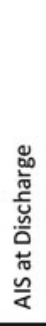 & Methodology Notes \\
\hline \multirow{7}{*}{ 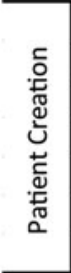 } & Age at Injury & & & & & & & & & & & & & & & Kernel Density Fitted \\
\hline & Gender & & & & & & & & & & & & & & & Frequency Table \\
\hline & Distance from Injury to DSCIC & $\checkmark$ & & & & & & & & & & & & & & Frequency Table \\
\hline & Mechanism of Injury & $\checkmark$ & $\checkmark$ & & & & & & & & & & & & & Multinomial Logistic Regression \\
\hline & Energy of Injury & $\checkmark$ & & & $\checkmark$ & & & & & & & & & & & Multinomial Logistic Regression \\
\hline & Neurological Level & $\checkmark$ & $\checkmark$ & & $\checkmark$ & $\checkmark$ & & & & & & & & & & Multinomial Logistic Regression \\
\hline & ASIA Impairment Scale (AIS) at Admission & $\checkmark$ & $\checkmark$ & & $\checkmark$ & $\checkmark$ & $\checkmark$ & & & & & & & & & Ordinal Logistic Regression \\
\hline \multirow{3}{*}{ 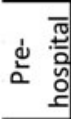 } & Go to $\mathrm{DSCIC}$ & & & $\checkmark$ & & & $\checkmark$ & & & & & & & & & Binomial Logistic Regression \\
\hline & Direct to DSCIC & & & $\checkmark$ & & & & & & & & & & & & Frequency Table \\
\hline & Time to DSCIC Arrival & & & $\checkmark$ & $\checkmark$ & $\checkmark$ & & $\checkmark$ & & $\checkmark$ & & & & & & Lognormal Fitted \\
\hline \multirow{5}{*}{ 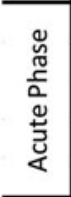 } & \begin{tabular}{|l} 
Surgery \\
\end{tabular} & $\checkmark$ & & & & & $\checkmark$ & $\checkmark$ & & & & & & & & Frequency Table \\
\hline & Complications & $\checkmark$ & & & & & $\checkmark$ & $\checkmark$ & & & & & & & & Subject Matter Experts \\
\hline & Mortality & $\checkmark$ & & & & $\checkmark$ & $\checkmark$ & $\checkmark$ & & & & & & & & Binomial Logistic Regression \\
\hline & Acute Length of Stay & $\checkmark$ & & $\checkmark$ & $\checkmark$ & & $\checkmark$ & $\checkmark$ & & $\checkmark$ & $\checkmark$ & $\checkmark$ & & & & Linear Regression \\
\hline & Acute Discharge Destination & $\checkmark$ & & & & & & $\checkmark$ & & & & & & & & Logistic Regression \\
\hline \multirow{5}{*}{ 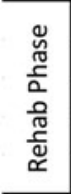 } & Go to Rehab & $\checkmark$ & & & & & & $\checkmark$ & & & & & $\checkmark$ & & & Binomial Logistic Regression \\
\hline & FIM at Admission & $\checkmark$ & & & & & $\checkmark$ & $\checkmark$ & & & & & & & & Linear Regression \\
\hline & FIM at Discharge & $\checkmark$ & & & & & $\checkmark$ & & & & & & & $\checkmark$ & $\checkmark$ & Linear Regression \\
\hline & AIS at Discharge & $\checkmark$ & & & & $\checkmark$ & & $\checkmark$ & & & & & & & & Ordinal Logistic Regression \\
\hline & Rehab Length of Stay & & & & & & $\checkmark$ & $\checkmark$ & $\checkmark$ & $\checkmark$ & & & $\checkmark$ & & & Linear Regression \\
\hline
\end{tabular}

FIG. 2. Summary of DES variables estimated and methodology involved in creating the PCM of the ACT Model (adapted and reprinted from PLOS ONE, Volume 8, 20138). DES, Discrete Event Simulation; PCM, Processes of Care Model; ACT, Access to Care and Timing; DSCIC, designated spinal cord injury centre; FIM, Functional Independence Measure. 


\section{Model Development}

\section{Model design}

The ACT Model simulates the provision of pre-hospital, acute, and rehabilitation services to persons with tSCI in Canada (Fig. 1). Conception of the model design included persons with SCI and other stakeholders with expertise in operations research, clinical research, health administration, medicine, surgery, rehabilitation, community health, and consumer engagement. As many of these stakeholders operate in one particular phase of care, the ACT Model provides the opportunity to view their contribution to healthcare delivery as part of an integrated system. This integrated view reflects the patient perspective as patients transition or flow through multiple phases of the care continuum. End users were involved early in the process of model development, which was essential in ensuring the simulation will meet the needs of end users and aiding in identification of possible errors in assumptions and patient flows. ${ }^{9}$

\section{Validation and reporting}

The ACT Model was validated using standard statistical validation for individual estimations, comparison of model outputs with data from the Rick Hansen SCI Registry (RHSCIR), ${ }^{10}$ and verification of patient flows and outputs with site personnel and SCI experts. The RHSCIR is a prospective, longitudinal patient registry of individuals with a new tSCI in Canada. ${ }^{10}$ A validation meeting with the original stakeholders also was held to explain the methodology employed to develop the model and to provide a detailed review of the model outputs. Methodology and results of the model also have been published in a number of peer-reviewed articles. ${ }^{6-8}$ Iterative and continuous model validation will be done to keep the model up to date and relevant.

\section{Maintenance and upkeep}

The ACT Model is data intensive, and due to the relatively low incidence of tSCI, the heterogeneity of tSCI, and the challenges with data availability, the current scope of the model is limited and can be enhanced. Consequently, in instances where data from the RHSCIR or other databases were not available, results from the literature were used to supplement the model, which may reduce relevance and specificity of some model outputs. An update to the model with the latest RHSCIR dataset comprising additional years of data is currently being planned.

\section{Methodology}

In the ACT Model, the DES simulates individuals with tSCI from injury through to rehabilitation in the PCM. Then, as individuals are discharged into the community, they enter the HPM where long-term outcomes and costs are assessed and calculated (Fig. 1). Data sources populating the model were the RHSCIR and process maps describing the RHSCIR acute and rehabilitation facilities, supplemented by literature review, subject matter experts, and other data sources including the National Trauma Registry and the National Rehabilitation Reporting System — of which both are from the Canadian Institute for Health Information.

Generalized linear models, including multiple linear regression and logistic regression analysis, were used to estimate the effect of demographic and injury attributes on outcomes in the DES,

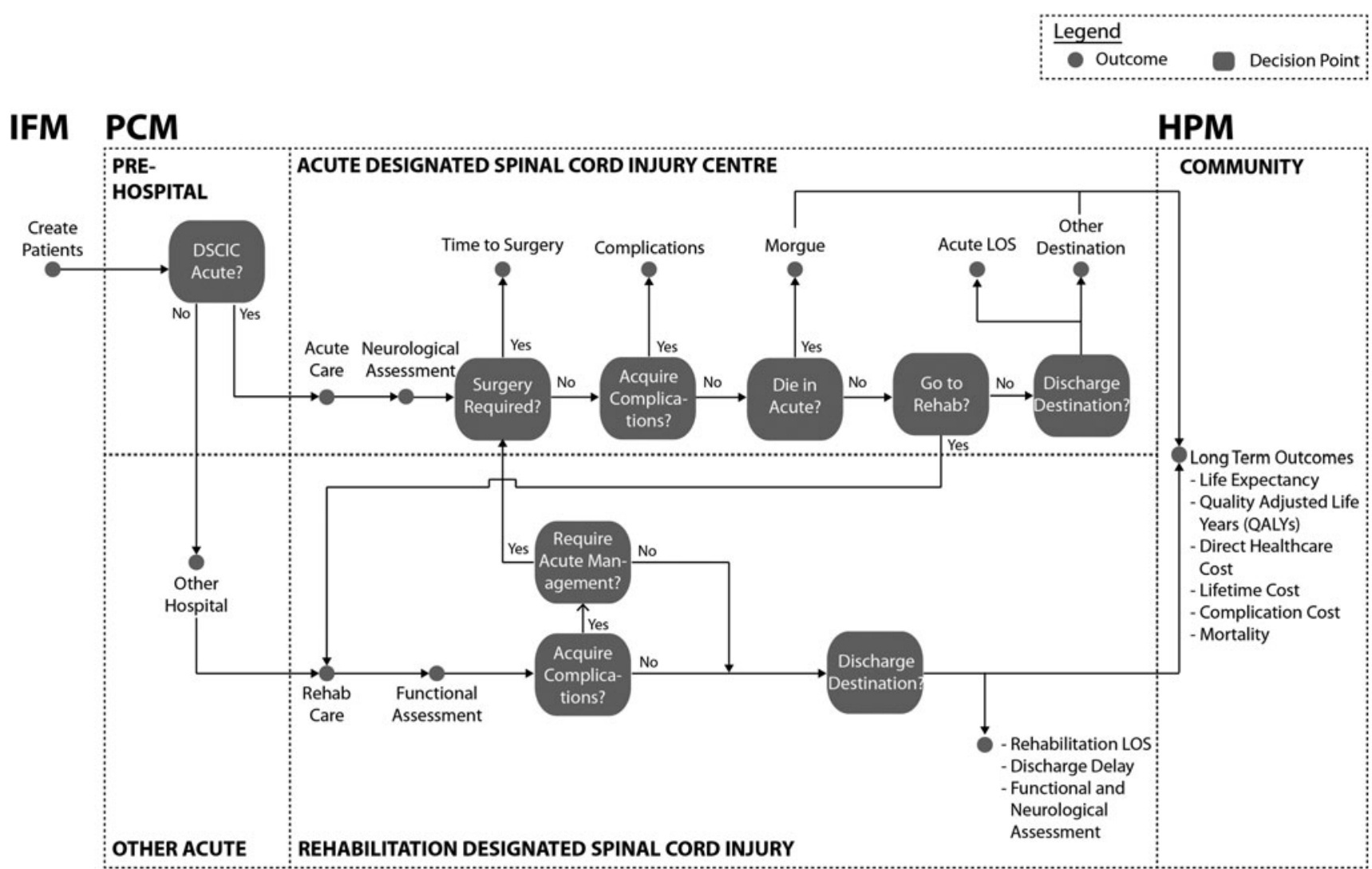

FIG. 3. Illustration of the ACT Model decision tree for flow of patients with traumatic spinal cord injury from point of injury to community reintegration. Multivariate regression analyses determine yes/no at decision points. ACT, Access to Care and Timing; IFM, Incidence Forecasting Model; PCM, Processes of Care Model; HPM, Health Progression Model; DSCIC, designated spinal cord injury centre; LOS, length of stay. 


\begin{tabular}{|c|c|c|c|c|c|c|c|c|c|c|c|c|c|c|c|c|c|}
\hline 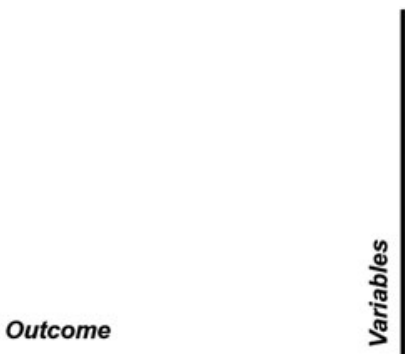 & ळ & 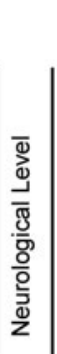 & $\frac{\infty}{\mathbb{L}}$ & 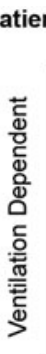 & 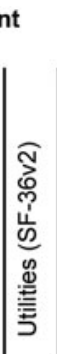 & 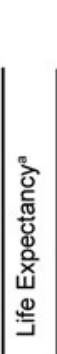 & 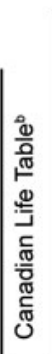 & 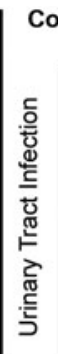 & $\begin{array}{l}\frac{5}{\mathrm{C}} \\
\mathrm{Q}\end{array}$ & 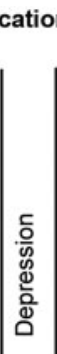 & 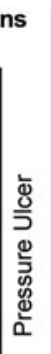 & 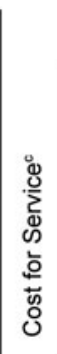 & 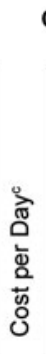 & 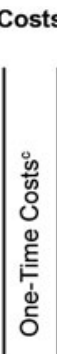 & 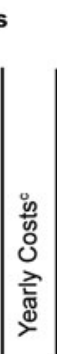 & 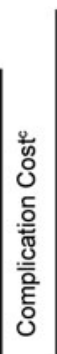 & Methodology Notes \\
\hline Life Expectancy & $\checkmark$ & $\checkmark$ & $\checkmark$ & $\checkmark$ & & $\checkmark$ & & & & & & & & & & & 3rd Order Polynomial Fit \\
\hline Quality Adjusted Life Years (QALYs) & & & & & $\checkmark$ & & & $\checkmark$ & $\checkmark$ & $\checkmark$ & & & & & & & Multivarite Logistic Regression \\
\hline Direct Healthcare Costs & & $\checkmark$ & & & & & & & & & & $\checkmark$ & $\checkmark$ & & & & Polynomial Equation \\
\hline Lifetime Costs & & $\checkmark$ & $\checkmark$ & & & $\checkmark$ & & & & & & & $\checkmark$ & $\checkmark$ & & & Polynomial Equation \\
\hline Complication Costs & & & & & & & & $\checkmark$ & & & $\checkmark$ & & & & & $\checkmark$ & Polynomial Equation \\
\hline Mortality & & & & & & & $\checkmark$ & & & & & & & & & & Cox Proportional Hazards Model ${ }^{d}$ \\
\hline
\end{tabular}

FIG. 4. Summary of HPM variables estimated and methodology involved in creating the HPM of the ACT Model. ${ }^{\mathrm{a} N a t i o n a l ~ S p i n a l}$ Cord Injury Statistical Centre (2009) ${ }^{11}$; ${ }^{\mathrm{b}}$ Statistics Canada (2006) ${ }^{12}$; ${ }^{\mathrm{C}}$ Krueger and colleagues $(2013){ }^{13}$; ${ }^{\mathrm{K}}$ Krause and colleagues $(2009) .{ }^{14}$ HPM, Health Progression Model; ACT, Access to Care and Timing; SF-36v2, the Medical Outcome Study 36-Item Short Form Version 2 questionnaire.

depending on the distribution of response variables and their statistical properties (Fig. 2). Age- and gender-specific incidence of tSCI input in the DES can be taken from RHSCIR data or from predictions calculated from the IFM. Based on the incidence, the DES creates simulated individuals with tSCI with attributes such as date and time of injury, mechanism of injury, neurological injury severity and level, and injury energy. Decision protocols, such as pre-hospital protocols that determine whether patients are directly admitted to specialized acute care facilities, also are applied to the simulated cohort. As the simulated individuals with tSCI reach specialized acute care and transition through to rehabilitation, they consume resources and accumulate properties such as therapeutic episodes (e.g., surgery), length of stay, secondary complications, and changes to neurological impairment (Fig. 3). A powerful feature of the model is that these properties, or outcomes, can be assessed in comparison with system outcomes, such as total direct care cost, to evaluate the net effect on the healthcare system.

Long-term outcomes, such as health-related outcomes, quality adjusted life-years, and healthcare costs, are calculated in the HPM, which tracks the health and economic outcomes of simulated individuals after exiting the healthcare system and through their rest of life (Fig. $4^{11-14}$ ). Integration of the PCM and HPM allows for the

Table 1. "What If" Simulation Scenarios Analyzed with the ACT Model Described in This Focus Issue

Topic Scenario(s) Outcome of interest

Geomapping and triage of tSCI (Cheng and colleagues)

Rehabilitation intensity (Truchon and colleagues)

Insights into LOS (Burns and colleagues)

Resource planning (Ahn and colleagues)
What if all patients who were injured within $40 \mathrm{~km}$ of a RHSCIR acute facility were triaged directly to a RHSCIR facility?

What if all patients who were injured within $40 \mathrm{~km}$ of a RHSCIR acute facility were triaged indirectly to a RHSCIR facility?

What if the intensity of rehabilitation therapy was increased by $50 \%$ or $100 \%$ ?

What if patient populations were switched between acute facilities?

What will be the effect on healthcare financial resources with the projected change in tSCI incidence over the next 20 years?
Patient: $n / a$

System: Time to RHSCIR facility arrival

Long-term: $\mathrm{n} / \mathrm{a}$

Patient: $\mathrm{n} / \mathrm{a}$

System: Motor FIM efficiency, rehabilitation LOS, bed utilization, direct healthcare costs

Long-term: n/a

Patient: $n / a$

System: Time to surgery, acute and rehabilitation

LOS, direct healthcare costs

Long-term: Lifetime care costs

Patient: Inhospital mortality

System: Acute admissions, acute and rehabilitation LOS, direct healthcare costs

Long-term: QALYs, life expectancy, life years lost, lifetime healthcare costs

ACT, Access to Care and Timing; tSCI, traumatic spinal cord injury; RHSCIR, Rick Hansen Spinal Cord Injury Registry; n/a, not applicable; LOS, length of stay; FIM, Functional Independence Measure; QALYs, quality-adjusted life years. 
evaluation, from a system level perspective, of the effect of intervention on patient and system outcomes in each phase of care and the rest of the individual's life.

\section{Application of the ACT Model}

The development of and the outputs from the ACT Model have been applied in this focus issue of the Journal of Neurotrauma to describe the processes of care delivery after tSCI in Canada, evaluate relationships between patient and system factors on outcomes, quantify the economic impact of secondary complications, forecast future system loads, identify gaps in knowledge, and address organizational policy questions. The impact of hypothetical policy/practice changes or interventions can be evaluated by running "what if" scenarios through the ACT Model (Table 1) by adjusting the values at a specific decision point (Fig. 3).

Because the model simulates the continuum of care as an interrelated system, the direct and indirect impacts of implementing policy/ practice changes can be quantitatively estimated for a particular phase of care, or in upstream or downstream phases. Hypothetical clinical or administrative interventions can be tested for cost effectiveness using the ACT Model before initiation of any implementation projects save time and cost. By providing a predictive link between interventions and outcomes, the ACT Model also can facilitate the use of system level indicators to measure quality of care and inform policy/practice changes as described in Fehlings and colleagues in this focus issue.

In addition to the intended application of the model, the rigorous data analysis during development of this data-intensive model revealed opportunities to improve measuring and reporting of data and led to recommendations to further enhance data collection and standardization for the RHSCIR. As explained in Dvorak and colleagues in this focus issue, these recommendations are applicable to other registries and will help to further research in advancing care.

\section{Conclusion}

The ACT Model can assist effective translation of research knowledge to practice by providing an opportunity to test policies and interventions on a simulated cohort of patients with tSCI and to determine impacts to both the patient and the healthcare system. The methodologies employed in this model provide a framework to look into the complexity of interactions within and among the different SCI programs, sites and phases of care.

\section{Acknowledgements}

The authors thank the RHSCIR Network and all of the participating RHSCIR facilities: Vancouver General Hospital, GF Strong Rehabilitation Centre, Foothills Medical Centre, Royal Alexandra Hospital, University of Alberta Hospital, Glenrose Rehabilitation Hospital, Royal University Hospital, Saskatoon City Hospital, Winnipeg Health Sciences Centre, St. Michael's Hospital, Sunnybrook Health Sciences Centre, Toronto Western Hospital, Toronto Rehabilitation Institute, Hamilton General Hospital, Hamilton Health Sciences - Regional Rehabilitation Centre, University Hospital (London), Victoria Hospital (London), Parkwood Hospital (London), The Ottawa Hospital - Civic Campus, The Ottawa Hospital Rehabilitation Centre, Hôpital de l'Enfant Jésus, Institut de réadaptation en déficience physique de Québec, Hôpital du SacréCoeur de Montréal, Centré de réadaptation Lucie-Bruneau, Institut de réadaptation Gingras-Lindsay-de-Montréal, QEII Health Sciences Centre, Nova Scotia Rehabilitation Centre, Saint John Regional Hospital, Stan Cassidy Centre for Rehabilitation, St. John's Health Sciences Centre and Dr. Leonard A. Miller Rehabilitation Centre.

\section{Author Disclosure Statement}

This study was supported by financial contributions from the Rick Hansen Institute, the Ontario Neurotrauma Foundation and the Government of Canada through Health Canada and Western Economic Diversification Canada.

\section{References}

1. Easterbrook, P.J., Doherty, M.C., Perrie, J.H., Barcarolo, J.L., and Hirnschall, G.O. (2014). The role of mathematical modelling in the development of recommendations in the 2013 WHO consolidated antiretroviral therapy guidelines. AIDS 28, S85-S92.

2. The HIV Modelling Consortioum Department of Infections Disease Epidemiology. HIV Modelling Consortium. Available at: www. hivmodelling.org. Accessed March 12, 2017.

3. Tappenden, P., Chilcott, J., Brennan, A., Squires, H., and Stevenson, M. (2012). Whole disease modeling to inform resource allocation decisions in cancer: a methodological framework. Value Health 15, 1127-1136.

4. Marshall, D.A., Burgos-liz, L., Eng, I., Ijzerman, M.J., Osgood, N.D., Padula, W.V, Higashi, M.K., Wong, P.K., Pasupathy, K.S., and Crown, W. (2015). Applying dynamic simulation modeling methods in health care delivery research-The SIMULATE checklist: Report of the ISPOR Simulation Modeling Emerging Good Practices Task Force. Value Health 18, 5-16.

5. Marshall, D.A., Burgos-liz, L., Eng, I., Ijzerman, M.J., Crown, W., Padula, W.V, Wong, P.K., Pasupathy, K.S., Higashi, M.K., and Osgood, N.D. (2015). Selecting a dynamic simulation modeling method for health care delivery research-Part 2: Report of the ISPOR Dynamic Simulation Modeling Emerging Good Practices Task Force. Value Health 18, 147-160.

6. Noonan, V.K., Soril, L., Atkins, D., Lewis, R., Santos, A., Fehlings, M.G., Burns, A.S., Singh, A., and Dvorak, M.F. (2012). The application of operations research methodologies to the delivery of care model for traumatic spinal cord injury: the access to care and timing project. J. Neurotrauma 29, 2272-22782.

7. Atkins, D., Noonan, V.K., Santos, A., Lewis, R., Fehlings, M., Burns, A., and Dvorak, M. (2012). Secondary complications in SCI across the continuum: Using operations research to predict the impact and optimize management strategies. Top. Spinal Cord Inj. Rehabil. 18, 57-66.

8. Santos, A., Gurling, J., Dvorak, M.F., Noonan, V.K., Fehlings, M.G., Burns, A.S., Lewis, R., Soril, L., Fallah, N., Street, J.T., Bélanger, L., Townson, A., Liang, L., and Atkins, D. (2013). Modeling the patient journey from injury to community reintegration for persons with acute traumatic spinal cord injury in a Canadian centre. PLoS One 8, e72552.

9. Lehaney, B., and Hlupic, V. (1995). Simulation modelling for resource allocation and planning in the health sector. J. R. Soc. Promot Health 115, 382-385.

10. Noonan, V.K., Kwon, B.K., Soril, L., Fehlings, M.G., Hurlbert, R.J., Townson, A., Johnson, M., and Dvorak, M.F. (2012). The Rick Hansen Spinal Cord Injury Registry (RHSCIR): a national patientregistry. Spinal Cord 50, 22-27.

11. National Spinal Cord Injury Statistical Center. (2009). Annual Report for the Spinal Cord Injury Model Systems. Birmingham, Available at: www.nscisc.uab.edu/PublicDocuments/reports/pdf/2009\%20NSCISC $\% 20$ Annual\%20Statistical\%20Report \%20-\%20Complete\%20Public\% 20Version.pdf. Accessed March 12, 2017.

12. Statistics Canada. (2006). Life tables, Canada, provinces and territories 2000-2002. Statistics Canada. Availabe at: http://publications.gc .ca/collections/Collection/Statcan/84-537-X/84-537-XIE2006001.pdf. Accessed March 12, 2017.

13. Krueger, H., Noonan, V.K., Trenaman, L.M., Joshi, P., and Rivers, C.S. (2013). The economic burden of traumatic spinal cord injury in Canada. Chronic Dis. Inj. Can. 33, 113-122.

14. Krause, J.S., Zhai, Y., Saunders, L.L., and Carter, R.E. (2009). Risk of mortality after spinal cord injury: an 8-year prospective study. Arch. Phys. Med. Rehabil. 90, 1708-1715.

\author{
Address correspondence to: \\ Vanessa K. Noonan, PhD, PT \\ Rick Hansen Institute \\ 6400-818 West 10th Avenue \\ Vancouver, British Columbia \\ V5Z 1 M9 Canada
}

E-mail: vnoonan@rickhanseninstitute.org 